\title{
Gender: Diskurse und Perspektiven für die Psychotherapie
}

\author{
Brigitte Schigl · Leonore Lerch
}

Geschlecht und Geschlechtlichkeit sind in der Psychotherapie seit ihren Anfängen Thema. Die Wirkung von Geschlechtskonstruktionen und -zugehörigkeiten im Sinne von Gender wurden allerdings in den meisten Psychotherapieverfahren nur wenig beschrieben. Geschlecht wird sozial auf vielfältige Weise hergestellt, und schafft Differenz, Ungleichheit, Hierarchien und Benachteiligungen. Nach über 100 Jahren internationaler Frauenbewegungen und Frauenwahlrecht sowie 50 Jahren feministischer Psychotherapie sollte man meinen, dass Gender auch im Mainstream der Psychotherapie-Praxis angekommen ist.

Als Herausgeberinnen dieses Heftes mussten wir feststellen: Dies ist (leider) nur teilweise so.

In der Planung des Heftes wurden wir von verschiedenen Seiten mit abwehrenden Reaktionen konfrontiert, z. B. dass das Thema Gender nicht mehr aktuell und zeitgemäß sei, dass die Verwendung einer gendergerechten Sprache die Lesbarkeit der Artikel erschwere, ja sogar verunmögliche, dass geschlechtsspezifische Unterschiede und soziale Verortungen in der Psychotherapie keine Rolle spielten, da wir sowieso immer nur auf individueller Ebene mit der Person arbeiten könnten, die in der Therapie vor uns sitze.

Nach diesen Erfahrungen im Vorfeld erscheint es uns umso wichtiger, Gender im Kontext von psychotherapeutischer Theorie und praktischem Arbeiten zu beleuchten und Be-Handlungsempfehlungen für die Psychotherapie abzuleiten.

Im vorliegenden Heft ist es uns gelungen, unterschiedliche Aspekte von Gender in den Fokus zu rücken und ihre Bedeutung für die Psychotherapie

\section{B. Schigl $\cdot$ L. Lerch $(\bowtie)$}

psychotherapie forum, Österreichischer Bundesverband für Psychotherapie (ÖBVP), Löwengasse 3/3/4, 1030 Wien, Österreich

oebvp.psychotherapieforum@psychotherapie.at aufzuzeigen. Wir folgen dabei den Diskursen, wie sie historisch gewachsen ineinandergreifen und haben versucht, die Beiträge entsprechend dieser Entwicklung $\mathrm{zu}$ ordnen. Die Autor_innen schlagen dabei einen Bogen, der mit der Geschichte des GenderDenkens als feministischem Anspruch in der Psychotherapie beginnt. Überlegungen zur gesellschaftlichen Konstruktion von Gesundheit und Krankheit folgen. Doing Gender als Phänomen in psychotherapeutischen Prozessen und die Frage nach einer geschlechtergerechten Therapie in der Behandlung von Paaren zeigen die praktische Relevanz der Gender Diskurse in der Psychotherapie. Im Anschluss werden Dimensionen männlicher Sozialisation in einer patriarchalen Gesellschaft beleuchtet. Entlang des Prozesses der Entwicklung der Gender Theories stehen sodann Aspekte der Therapie homosexueller Patient_innen im Zentrum der Betrachtung und stellen die rezenten Queer Theories und die Diskurse um Diversity in den Mittelpunkt. Dabei beschäftigen sich die Autor_innen mit Trans- und Intergeschlechtlichkeit sowie den Intersektionen, die sich aus verschiedenen Aspekten von Diversity auch in der Psychotherapie abbilden.

Dies sind die Beiträge im Einzelnen:

Nora Ruck, Maria Köhne, Max Beck, Florian Knasmüller, Vera Luckgei und Elisabeth Parzer geben einen Abriss der historischen Entwicklung von feministischen, frauenspezifischen und gendersensiblen Therapieansätzen, die sich in den meisten westlichen Ländern seit Anfang der 1970er entwickelten. Sie wurzeln in sogenannten consciousness-raising Gruppen und stellen eine Querschnittperspektive dar, die unter Einbezug gesellschaftlicher Strukturen und Bedingungen darauf abzielen, bestehende psychotherapeutische Theorien und Methoden zu ergänzen.

Bettina Zehetner zeigt anhand diverser Störungsbilder, wie die Anpassung an überfordernde Verhältnis- 
se und widersprüchliche Geschlechternormen krank machen können und wie umgekehrt Krankheit als Verweigerung von Anpassung durchaus ein Zeichen psychischer Gesundheit sein kann. Geschlechtsspezifische Krankheitsformen beinhalten darum auch kritisches Potenzial, indem sie das Leiden an Geschlechternormen demonstrieren.

Brigitte Schigl umreißt die Prozesse, in denen Doing Gender in der Psychotherapie wirksam wird. Dabei wird der Blick auf Therapeut_innen wie Patient_innen gerichtet, die beide Gender in ihren Interaktionen herstellen. Zur Erörterung werden qualitative Daten von Psychotherapeut_innen sowie quantitative Daten aus einer großen Patientenbefragung (RISK, Donau Universität Krems) verwendet und je nach Zusammensetzung der therapeutischen Dyade spezifische Situationen und Themen im therapeutischen Prozess analysiert.

Sabine Kirschenhofer lädt zu einer Auseinandersetzung mit Geschlecht und Geschlechterverhältnissen ein und wählt dafür das Arbeitsfeld der Paartherapie mit heterosexuellen Paaren. Nach einer Begriffsklärung, was ,geschlechtergerecht' im Kontext von Paartherapie für sie bedeutet, wirft sie aus einer feministischen Perspektive einen kritischen Blick auf zeitgenössische patriarchale Verhältnisse in der Paartherapie. Dabei prüft sie anhand eines Fallbeispiels, wie die systemische Therapie für dieses Thema nutzbar gemacht werden kann und wo sie versagt.

Lothar Böhnisch beschreibt biografische Stationen (frühe Kindheit, Pubertät, Adoleszenz und Integration ins Erwachsenenalter), die Männer auf dem Weg ihrer Sozialisation erfahren. Er zeigt aus einer tiefenpsychologischen Perspektive auf, dass trotz veränderter Rollenmodelle von Männlichkeit und Weiblichkeit ein Mechanismus der Idolisierung des Männlichen wirksam ist, der vor allem in männlichen Gruppendynamiken freigesetzt wird.

Claudia Höfner, Kai Ginkel und Gerald Käfer-Schmid befassen sich mit der Sozialisation homo- und bisexueller Menschen, die innerhalb der heteronormativen Mehrheitsgesellschaft einige Besonderheiten birgt, die in Bezug auf die therapeutische Beziehung reflektiert werden müssen. Hierbei steht als Zielsetzung der Einsatz für eine bessere, barriere- und diskriminierungsarme Gesundheitsversorgung und Behandlung durch kompetente und wertschätzende Fachkräfte im Mittelpunkt.

Esther Hutfless sieht die Notwendigkeit, über gleichstellungspolitische Ansätze hinauszugehen, da diese die Identitätskategorien reproduzieren und fortschreiben. Sie regt an, die Norm selbst in Frage zu stellen bzw. zu dekonstruieren und zeigt auf, inwiefern insbe- sondere queere Ansätze als produktive und kritische Interventionen in psychotherapeutischen Diskursen und Praxen wirken können.

Leonore Lerch gibt eine Einführung in die Ansätze von diversity \& intersectionality und unterstreicht ihre Bedeutung für die Psychotherapie, insbesondere den Perspektivenwechsel hin $\mathrm{zu}$ einer globalen Psychotherapie. Unter Bezugnahme auf ein Selbstverständnis der Psychotherapie als Menschenrechtsprofession plädiert sie für die Entwicklung einer differenz- und dominanzkritischen psychotherapeutischen Haltung.

Cornelia Kunert diskutiert die Bedeutung und den Vorrang der Erste-Person-Perspektive für die Psychotherapie mit Transgender Personen. Sie betrachtet die Zuschreibungen der Geschlechtsidentität aufgrund von anatomischen Verhältnissen des Körpers oder anderen biologischen Daten, als auch die Bezugnahme auf neurologische Erkenntnisse zum Zwecke der Zuordnung zu einem Geschlecht als biologistischen Reduktionismus - zu Lasten der Variabilität des Selbstmodells und der Pluralität der subjektiven Erfahrung des Seins.

Lisa Wanner und Finn* L. Landsteiner befassen sich mit Fragen zu vielfältigen Geschlechtlichkeiten in psychotherapeutischen Räumen und erörtern die ambivalente Rolle von Psychotherapeut_innen als Vertreter_innen eines machtstützenden Systems, das gleichzeitig individuelle Selbstbestimmung stärken soll. Aus ihren Überlegungen leiten sie spezifische Bestärkungs- und Gefahrenpotentiale psychotherapeutischer Räume und Reflexionsebenen für Psychotherapeut_innen ab.

Die Originalarbeiten und Übersichtsartikel werden ergänzt durch zum Themenschwerpunkt passende Buchrezensionen.

Wir haben den Autor_innen freigestellt, welche Art der gendergerechten Sprache bzw. Schreibweise sie bevorzugen - so bilden die Texte auch verschiedene Aspekte dieser Bemühungen ab. Die Uneinheitlichkeit ist gewollt und macht Vielfalt auch hier sichtbar.

Brigitte Schigl \& Leonore Lerch

Verantwortliche Editorinnen in Chief für die Doppelausgabe Gender: Diskurse und Perspektiven für die Psychotherapie

Interessenkonflikt B. Schigl und L. Lerch geben an, dass kein Interessenkonflikt besteht.

Hinweis des Verlags Der Verlag bleibt in Hinblick auf geografische Zuordnungen und Gebietsbezeichnungen in veröffentlichten Karten und Institutsadressen neutral. 